\title{
Análisis descriptivo sobre la función de la contabilidad en 21 empresas del Gran Concepción (Chile)
}

\author{
MARCELO NAVARRETE ESPARZA* \\ JAIME LANDAETA BAHAMONDES** \\ MARÍA SALAZAR AGUILERA***
}

* Magíster en Tributación. Académico del Departamento de Administración y Auditoría. Universidad del Bío Bío, Concepción, Chile. E-mail: m.navarrete@ubiobio.cl. ORCID: 0000-0003-4654-1139. Google Scholar: https://scholar.google.com/ citations?hl=es\&user=OYYzmpMAAAAJ.

** Magíster en Planificación y Gestión Tributaria. Académico del Departamento de Administración y Auditoría. Universidad del Bío Bío, Concepción, Chile. E-mail: jlandaet@ubiobio.cl. ORCID: 0000-0003-2487-7408. Google Scholar: https://scholar. google.com/citations?hl=es\&user=YTzKeKYAAAAJ.

*** Licenciada en Tecnología de la Información. Universidad del Bío Bío, Concepción, Chile. E-mail: mfsalazar@alumnos.ubiobio.cl. ORCID: 0000-0001-6896-1818. Google Scholar: https://scholar.google.com/ citations? user=20SMbw4AAAJ\&hl=es. 


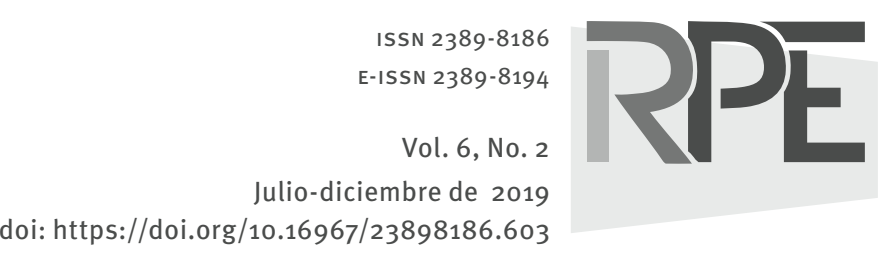

COMO CITAR ESTE ARTÍCULO How to cite this article:

Navarrete, M., Landaeta, J. y Salazar, M. (2019). Análisis descriptivo sobre la función de la contabilidad en 21 empresas del Gran Concepción (Chile). Revista Perspectiva Empresarial, 6(2), 74-86.

Recibido: 31 de mayo de 2019

Aprobado: 02 de octubre de 2019
RESUMEN El propósito de este trabajo es el de analizar descriptivamente la percepción de la administración respecto a la función de la contabilidad en las decisiones de la organización en empresas pertenecientes a los sectores económicos más importantes del Gran Concepción (Chile). Se analizó empíricamente 21 respuestas recibidas de distintas empresas. La investigación utilizó un enfoque cuantitativo con base en una medición numérica y análisis estadístico. El alcance del trabajo fue de carácter exploratorio dado que es una situación poco estudiada en Chile. Los resultados indican que en gran parte de las respuestas de la administración se percibe de manera negativa a la función de la contabilidad. La reflexión de estos resultados apunta a que la percepción de la administración vislumbra que la función de la contabilidad debe transformarse en una herramienta de apoyo a su gestión a través de una función que tienda a generar una línea de especialización en contabilidad.

PALABRAS CLAVE contabilidad, función, administración, formación profesional, gestión, percepción.

\section{Descriptive analysis on the role of accounting in 21 companies of Gran Concepción (Chile)}

ABSTRACT The aim of this work is to descriptively analyze the perception of management with respect to the role of accounting in organizational decisions of firms belonging to the most important economic sectors of Gran Concepción (Chile). 21 responses received from different companies were empirically analyzed. This research used a quantitative approach based on a numerical measurement and a statistical analysis. The scope of this work was of an exploratory nature given it is a rather unexplored situation in Chile. The results indicate that in most of the responses from the management, the role of accounting is negatively perceived. A reflection on these results points that the perception of management unveils that the role of accounting must be transformed into a tool to support its management through a role oriented to generate a specialization line in accounting.

KEYWORDS Accounting, role, administration, professional training, management, perception. 


\section{Análises descritivo sobre a função da contabilidade em 21 empresas da Grande Concepción (Chile)}

RESUMO 0 propósito deste trabalho é o de analisar descritivamente a percepção da administração com respeito à função da contabilidade nas decisões da organização em empresas pertencentes aos sectores económicos mais importantes da Grande Concepción (Chile). Se analisou empiricamente 21 respostas recebidas de distintas empresas. A investigação utilizou um enfoque quantitativo com base em uma medição numérica e análise estatístico. 0 alcance do trabalho foi de carácter exploratório dado que é uma situação pouco estudada no Chile. Os resultados indicam que em grande parte das respostas da administração se percebe de maneira negativa à função da contabilidade. A reflexão destes resultados aponta a que a percepção da administração vislumbra que a função da contabilidade deve transformar-se em uma ferramenta de apoio a sua gestão através de uma função que tende a gerar uma linha de especialização em contabilidade.

PALAVRAS-CHAVE contabilidade, função, administração, formação profissional, gestão, percepção. 


\section{Introducción}

La función de la contabilidad y de las personas que la ejercen dentro de una organización está directamente relacionada con la disciplina 'contable'. Esta es vista en muchas instancias limitada solo a actividades como la teneduría de libros, registros y aplicación de normas específicas.

A través del tiempo la contabilidad se ha ido fortaleciendo y complementando con otras disciplinas del saber tales como finanzas, economía, administración, legislación tributaria, auditoría. Prácticamente en todas estas áreas se puede distinguir un componente contable que beneficia al entendimiento y lenguaje de cada una de ellas. Las personas que estudian contabilidad deben ser capaces de comprender el lenguaje y uso práctico de esta disciplina, de manera que logren contribuir a satisfacer múltiples necesidades de información que administra una organización.

Subsisten múltiples necesidades de información generada de manera interna en cualquier tipo de organización (ya sea empresas, organismos públicos, ONG entre otras), en donde se observa un denominador común entre ellas. Esto tiene relación con la necesidad, por parte de la administración, de tomar decisiones que contribuyan al éxito de los objetivos establecidos por la entidad sobre la base de información interna/externa. La base de información interna está principalmente compuesta de contabilidad y la externa de todo aquello que proviene de fuentes regulatorias, cumplimiento medioambiental, leyes impositivas entre otras. Sin embargo tomar decisiones no es la única responsabilidad que tiene la administración puesto que también debe preparar y entregar, por ejemplo, informes, reportes, estadísticos de ventas, tanto a los dueños de la entidad como a los entes externos. Así, la gestión de los directivos requiere de profesionales especialistas que puedan comprender el lenguaje contable y transformarlo en una herramienta estratégica útil que contribuya a las buenas decisiones. Es así que, entonces, desde el punto de vista externo, la organización proporciona información hacia los distintos usuarios que consultan habitualmente información tal como el paquete de estados financieros. En este sentido es importante considerar el concepto de stakeholder (partes interesadas) asociado a una mirada de gobierno corporativo (Freeman, 1983). Estos entes externos a la organización poseen intereses sobre la empresa y constantemente observan aspectos como, por ejemplo, la estabilidad $y$ continuidad de sus operaciones en el tiempo.

La administración posee una percepción respecto a cuál es el aporte del área/departamento de contabilidad a los procesos de toma de decisiones que sellevan a cabo en una organización. Si la percepción de la administración es negativa o positiva, es una situación que puede generar expectativas distintas entre aquello que creen aportar los profesionales del área/departamento de contabilidad a la gestión en las decisiones de la organización y lo que cree la administración que esa área debería apoyar en su gestión. Específicamente, en nuestro caso, respecto a la función de la contabilidad en las decisiones de la organización es importante investigar cuál es la percepción de la administración superior (llámese gerencia, gobierno corporativo, subgerencia, generalmente mandos ejecutivos) en grandes empresas de la ciudad de Concepción (Gran Concepción).

\section{Revisión de literatura}

García (2004) indica que desde tiempos antiguos la contabilidad nació para efectos de satisfacer necesidades de información del hombre y la sociedad. Esta información ha ido evolucionando con el tiempo y a medida que surgieron nuevas necesidades de informar se requirió de una disciplina estructurada que pudiera satisfacerlas. Cada vez hace más sentido que la contabilidad genere más bondades que ciertas actividades de carácter normativo y/o de cumplimiento.

El uso que recibe la contabilidad y lo relevante de la información que genera debería ser considerado en todo proceso de toma de decisiones, este es el punto fundamental que acompaña esta investigación: encontrar la conexión entre la herramienta y su aplicabilidad a distintas operaciones que subsisten dentro de una organización y especialmente aquellas con fines de lucro como, por ejemplo, operaciones 
financieras, operaciones administrativas y operaciones de gestión, más allá de los aspectos básicos que podemos encontrar en ella.

Conectar a la contabilidad con el interés de la administración de la empresa ha sido un punto de reflexión para muchos autores, Cañibano (2004) escribe sobre la experiencia en España para empresas que cotizan en mercados de valores y se refiere a la importancia que existe entre la información contable y el gobierno de la empresa. El autor sostiene que la información contablefinanciera constituye el principal vehículo de comunicación entre los distintos entes internos y externos de la empresa, en especial del gobierno corporativo. Así las cosas, los autores de esta investigación concuerdan en ver a la contabilidad como una herramienta que deja de ser básica y que se transforma en un motor de información útil para interpretar lo que sucede dentro de la empresa.

Una postura similar es la de Dueñas (2007) que plantea que ha cambiado la contabilidad vista como un enfoque tradicional y de medición del beneficio. Respecto a este cambio, el mismo autor afirma lo siguiente: "esta última concepción está mucho más relacionada con las actuaciones de los agentes en un entorno complejo llamado mercado, y que por su naturaleza exige la toma adecuada de decisiones en hechos particularmente económicos" (p. 2). Los autores de esta investigación coinciden con ello dado que la contabilidad no se relaciona solo con registros básicos y el cumplimiento de normas sino que también debe responder a distintos tipos de entidades que se hallan en el mercado; de ahí que deba apoyar los procesos de toma de decisiones, rescatar información integral económica de la organización y al mismo tiempo cumplir un rol funcional y no neutral.

En cuanto a la integridad y funcionalidad de la contabilidad, Monterrey (1998) hace un estudio remontándose al nacimiento del movimiento positivista (contabilidad positiva) a objeto de conocer y explicar el mundo real de la contabilidad, de los contables y de las instituciones relacionadas.

Monterrey (1998) expresa una idea central interesante: la contabilidad debe entenderse como una parte integral de la economía, en interacción con otros ámbitos de esta, y que la información contable no es un fenómeno neutral, ya que afecta a la asignación de recursos y tiene consecuencias tanto en el ámbito de las organizaciones como en el de los mercados. (p. 32)

Otros autores, como Carrizo y León (2007), sostienen que "no es posible concebir a la Contabilidad como una disciplina que sólo intenta describir fenómenos, siempre el fin será el de utilizar información con algún propósito" (p. 4). Es decir que la contabilidad puede ser más que lo que en esencia representa para efectos de su uso práctico, a saber, apoyar -en complemento con otra información- a la administración día a día en la toma de decisiones.

Por su parte Buelvas y Mejía (2014) destacan el papel que ejerce la contabilidad de gestión dentro del sistema de información contable. La investigación desarrollada por estos autores es muy importante para este análisis descriptivo de la función de la contabilidad. Ellos subrayan en su escrito, el sentido que posee el rol de la contabilidad de gestión: "la contabilidad de gestión se constituye en el pilar para la toma de decisiones y así permanece en competencia en este mundo globalizado" (p. 2).

De acuerdo a la revisión de literatura efectuada hasta aquí, todos los autores coinciden en que la contabilidad va más allá de cumplir normas y registros. Las personas que conforman el área/departamento de contabilidad cumplen una función importante en el sentido que ella debe responder a las necesidades de los agentes externos e internos de la empresa, y esta función debe aportar a la administración para construir información clave para tomar sus decisiones.

A partir de los años 2008-2009, Chile inició un proceso de convergencia a nivel de la normativa contable vigente en ese momento. Antes de este proceso de convergencia, dicha información se utilizaba solo como marco normativo y de políticas contables adaptadas de las Normas Internacionales de Contabilidad -NIC-. Este significativo cambio contable implicó introducir un nuevo lenguaje a esta disciplina, lo que abrió 
el espacio a conceptos técnicos entre los que destacan: deterioro; revaluación; goodwill; instrumentos financieros y vida útil financiera. La convergencia en el país significó dar énfasis a conceptos contable-financieros que las NIC incluían dentro de su propósito principal, del mismo modo agregó valor al uso de este lenguaje haciéndolo más cercano y entendible a las necesidades de la administración a través de las distintas operaciones y/o transacciones diarias. Hasta antes de estos cambios en nuestro país, la contabilidad era vista solo como un marco de referencia y recibía un carácter de registro limitado a cumplimientos básicos de esta disciplina. Chile a través de distintos organismos reguladores como la Superintendencia de Bancos e Instituciones Financiera -SBIF-, la Comisión para el Mercado Financiero -CMF-, y otros entes reguladores del mercado financiero, hizo homogéneo este lenguaje internacional "contable-financiero" en todas las empresas del país; específicamente aquellas bajo regulación de las entidades fiscalizadoras, de las cuales la más grande corresponde a la CMF que incluye a unas 362 empresas en el país.

Es importante destacar que la contabilidad ha evolucionado en la forma de llevar sus registros de información desde sus orígenes. Hasta hace unas décadas atrás se registraba única y exclusivamente en registros de papel, hoy podemos observar la contabilidad electrónica y digital a través del uso de sistemas computacionales que son capaces de almacenar grandes cantidades de bases de datos contables; de igual manera sin dejar de mencionar también a la contabilidad virtual efectuada a través de bloques de cadena. A partir de esta información digital se pueden construir un sin límite de reportes, análisis financieros, análisis estadísticos, seguimiento y comportamientos operacionales que son necesarios para gestionar decisiones en cualquier organización. Tanto los autores expuestos como los que construyen esta investigación, coinciden en que la contabilidad es una herramienta útil capaz de interpretar los hechos económicos de una organización y transformarlos en información clave.

Por otra parte esta investigación usa el concepto 'percepción' como un medio a través del cual podemos conocer lo que opina la administración superior de una organización sobre la función de la contabilidad, por lo que tener entendimiento sobre la 'percepción' es muy importante para poder explicar este trabajo. Respecto a este concepto no se pretende hacer una profundización filosófica sobre esta palabra, lo que se intenta exponer es una idea base del vocablo para lograr posteriormente entender la palabra percepción y comprender qué elementos reúne.

Balmes (1848) nos presenta conceptos básicos sobre la percepción. Este autor desarrolla un capítulo especial para referirse a este tema; en primer lugar comienza explicando el concepto de 'entendimiento', el cual es la facultad de conocer. Luego agrega que el entendimiento está compuesto de tres actos: percepción, juicio y raciocinio. Así, para él: "la percepción es el acto con que conocemos la cosa, sin afirmar ni negar nada de ella. Juicio es el acto con el cual afirmamos o negamos una cosa de otra y raciocinio es el acto con el cual inferimos" (p. 28).

Al respecto, Amstrong (1966) afirma que: "la percepción no es nada más que la adquisición de conocimiento de, o, en ocasiones la adquisición de una inclinación a creer en, hechos particulares acerca del mundo físico, por medio de nuestros sentidos" (p. 131). Bajo el análisis de Armstrong se puede indicar que la percepción utiliza los sentidos de una persona sobre la base de un conocimiento del mundo físico. Más adelante en su obra se refiere a que la percepción proviene de una experiencia previa (conocimiento del mundo) y del uso de los sentidos (órganos sensoriales), ambas acciones constituyen los hechos particulares del mundo físico por medio de nuestros sentidos.

Por su parte Arnheim (1971) plantea que "la colaboración entre la percepción y el pensamiento en la cognición resultaría incomprensible si tal división existiera [...] son todas las operaciones mentales implicadas en la recepción, almacenaje y procesamiento de la información: Percepción sensorial, memoria, pensamiento, aprendizaje" (p. 27-28).

Aun cuando este autor asocia su obra al pensamiento visual, específicamente al arte visual, es importante destacar que la percepción de lo que se ve y observa guarda directa relación con el conocimiento que se tiene respecto a un objeto de estudio. 
Según Carterette y Friedman, la percepción es:

una parte esencial de la conciencia, es la parte que consta de hechos intratables y, por tanto, constituye la realidad como es experimentada. Esta función de la percepción depende de la actividad de receptores que son afectados por procesos provenientes del mundo físico. La percepción puede entonces definirse como el resultado del procesamiento de información que consta de estimulaciones a receptores en condiciones que en cada caso se deben parcialmente a la propia actividad del sujeto. (Como se citó en Arias Castilla, 2006, p. 9)

Así que una percepción simplista de la administración tiende a visualizar que la función de la contabilidad queda relegada solo a registrar, lo que puede implicar ausencia de conocimiento sobre esta disciplina y su funcionalidad dentro de la organización. Otras administraciones tienen una percepción más práctica y perciben que la función contable debería tender más a allá del registro contable y su transacción; es decir que esta área debería tender a involucrarse en la gestión del negocio y sus operaciones, aprovechando la fuente de información que se genera al interior de la contabilidad.

En este sentido Zeff (1985) ya se refería, en los años 80, a la evolución que había experimentado la contabilidad en Estados Unidos durante los últimos veinte años; allí la contabilidad pasó de un carácter normativo a una disciplina de utilidad para la toma de decisiones, capaz de proveer de información útil a distintos usuarios.

Por su parte Buelvas y Mejía (2014) exponen un tema muy relevante a partir de experiencias en Colombia. Para estos autores la contabilidad de gestión se convierte en una herramienta competitiva útil y atingente a estos nuevos tiempos de modernidad de negocios.

\section{Ellos sostienen que:}

en el ámbito empresarial, sus directivos se han dado cuenta que es imposible tomar decisiones contando sólo con la información que les brindan los estados financieros, saben que es relevante otra información complementaria de carácter financiero y no financiero que les brinda la contabilidad de gestión. (p. 2-3)

En tiempos modernos surge la necesidad de administrar grandes bases de datos conocidas hoy como big data. Es por ello que la función de la contabilidad pasa a transformarse en un núcleo generador de información importante para la empresa. Hasta aquí, resulta importante pensar que habría una necesidad que el profesional contable se especialice en un área específica como lo es la contabilidad de gestión.

En Chile, la contabilidad de gestión aún no está internalizada y más bien se ve como ajena a las actividades que normalmente desempeña un profesional de esta disciplina. No obstante, para la administración, esta área/departamento se ha transformado en una fuente de información relevante. Así pues, desde un punto de vista de gestión para la organización, ¿existirá una percepción negativa de la administración de grandes empresas dentro del Gran Concepción respecto a la función de la contabilidad? En este sentido el objetivo principal de este trabajo de investigación es hacer un análisis descriptivo sobre la base de la percepción de la administración respecto al impacto de la función contable en las decisiones de la organización, considerando que la administración posee una percepción negativa frente a la función del área/departamento de contabilidad.

\section{Metodología}

La presente investigación es de carácter empírico e intenta analizar de manera descriptiva la percepción que posee la administración respecto al impacto de la función contable en las decisiones de la organización, además considera la base de la revisión de literatura que se ha estudiado previamente.

La metodología utiliza un enfoque cuantitativo, ya que lleva a cabo una recolección de datos para probar la hipótesis con base en la medición numérica y el análisis estadístico con el propósito de analizar los patrones de comportamiento y 
probar teorías (Hernández, Fernández y Baptista, 2010).

Por otra parte, en primer lugar, el alcance dado a esta investigación fue exploratorio debido a que corresponde a una situación poco estudiada en el Gran Concepción; en segundo lugar utilizó un alcance correlacional para explicar la relación entre las variables definidas en esta investigación. En tercer lugar el trabajo también utilizó un corte transversal aplicable.

En este estudio se aplicó una encuesta (en adelante, "el instrumento") que consta de dos partes. La primera parte solicitó al encuestado su identificación a través de 13 preguntas; la segunda parte se enfocó en 31 preguntas que pretenden captar la percepción de la persona respecto a la investigación. El instrumento solo consideró respuestas cerradas de 5 puntos de acuerdo a la escala de Likert ${ }^{1}$. El instrumento fue construido sobre la base de indagaciones y/o consultas efectuadas a 3 ejecutivos escogidos al azar pertenecientes a las organizaciones.

Una vez confeccionado el instrumento se validó nuevamente con los mismos ejecutivos, pero ahora solicitando su retroalimentación y respuestas al mismo instrumento. Finalmente el instrumento fue dividido entre afirmaciones que son percibidas como negativas para la administración y afirmaciones que son percibidas como positivas por la misma.

\section{Población a estudiar}

De un total de 430 grandes empresas que existen en el Gran Concepción (Chile) se seleccionaron aleatoriamente 215 organizaciones para ser investigadas, de ellas solo 39 (población objetivo) tienen su contabilidad en el Gran Concepción ${ }^{2}$; posteriormente de las 39 , solo el 53,8 \% (21 empresas) de la población respondió el instrumento enviado por correo electrónico o aplicado en forma presencial. En la tabla 1 y en las figuras 1 y 2 se muestran los distintos datos de la población utilizada.

Tabla 1. Grandes empresas en Chile y en el Gran Concepción

\begin{tabular}{ll}
\hline \hline $\begin{array}{l}\text { Grandes empresas en Chile y en la } \\
\text { región del Biobío }\end{array}$ & $\begin{array}{l}\text { No. de } \\
\text { empresas }\end{array}$ \\
\hline \hline Chile & 14172 \\
\hline Región del biobío & 636 \\
\hline Gran Concepción & 430 \\
\hline Lota & 6 \\
\hline Coronel & 48 \\
\hline San Pedro de la Paz & 44 \\
\hline Santa Juana & 1 \\
\hline Concepción & 215 \\
\hline Chiguayante & 9 \\
\hline Hualqui & 4 \\
\hline Talcahuano & 59 \\
\hline Hualpén & 22 \\
\hline Penco & 15 \\
\hline
\end{tabular}

Fuente: Servicio de Impuestos Internos.

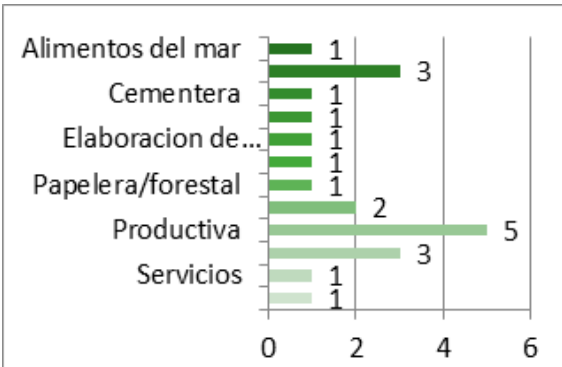

Figura 1. Las 21 empresas pertenecientes a distintos sectores económicos. Fuente: elaboración propia por parte de los autores.

\footnotetext{
1 Likert utilizado: (1) siempre; (2) casi siempre; (3) muchas veces; (4) muy pocas veces y (5) nunca.

2 Las 39 empresas con contabilidad en el Gran Concepción se obtuvieron luego de indagar telefónicamente o de manera personal en cada una de las 215 empresas seleccionadas aleatoriamente.
} 


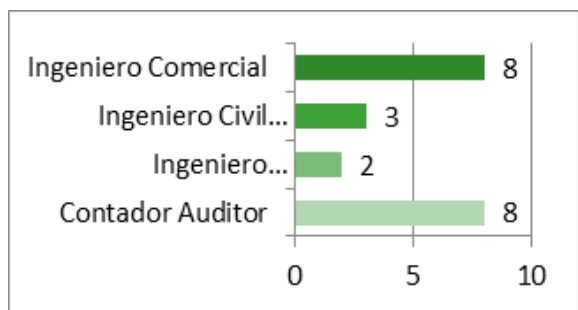

Figura 2. Ejecutivos que respondieron al instrumento Fuente: elaboración propia por parte de los autores.

\section{Metodología utilizada para analizar los resultados del instrumento}

Se recogieron los datos de las respuestas obtenidas a través del instrumento y enfocadas en las afirmaciones negativas que la administración percibe de la función de la contabilidad. En el instrumento hay 8 afirmaciones que responden a la categoría de percepción negativa de la función contable de acuerdo al número de la afirmación en la encuesta aplicada. La administración que respondió el instrumento está caracterizada por cargos. Luego se analizó la percepción negativa por medio de los distintos cargos a través del uso de la gráfica de radar, instrumento estadístico que nos permite una información descriptiva de los resultados obtenidos.

Con base en ello Pérez (2017) sostiene que estas gráficas facilitan las posibilidades de aplicación en distintos campos del conocimiento, siendo en este caso el de la percepción de la función de la contabilidad una ocasión importante para hacerlo. Las variables dependiente e independiente utilizadas en la gráfica de radar constan de la siguiente estructura: (i) variable dependiente, percepción del encuestado; (ii) variable independiente, cargo del encuestado.

\section{Resultados}

Las respuestas recibidas correspondieron a 21 grandes empresas 3 situadas dentro del Gran Concepción (octava región de Chile, provincia de Concepción). Las figuras 3 a la 7 muestran los gráficos que sustentan la hipótesis propuesta. Estas figuras se construyeron con el resultado del instrumento.

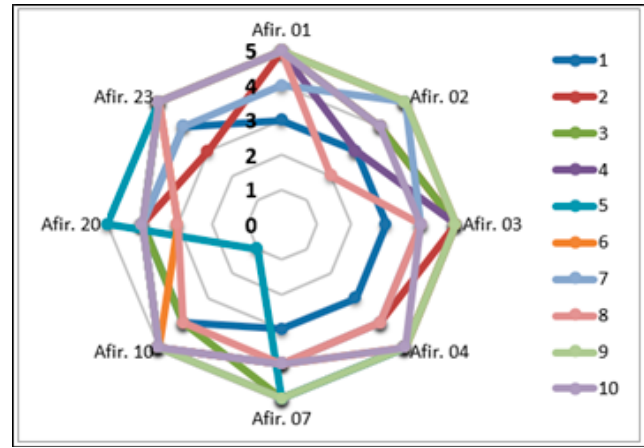

Figura 3. Muestra de las respuestas de los gerentes de administración y finanzas (10) sobre las afirmaciones negativas (8). Fuente: elaboración propia por parte de los autores.

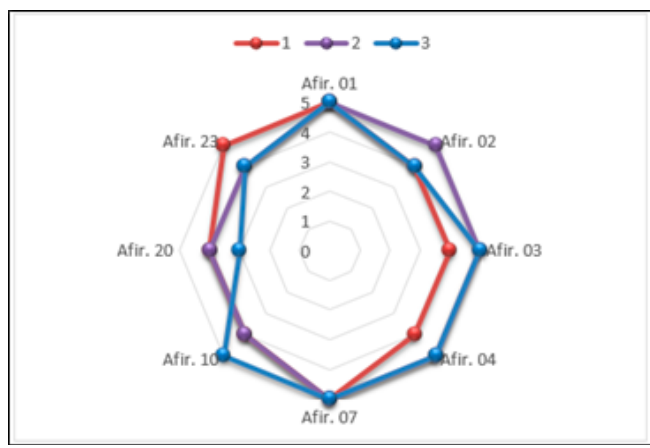

Figura 4. Muestra de las respuestas de los gerentes de finanzas y contabilidad (3) sobre las afirmaciones negativas (8). Fuente: elaboración propia por parte de los autores.

3 Estratificación de empresas en Chile, Ley 20.416. 


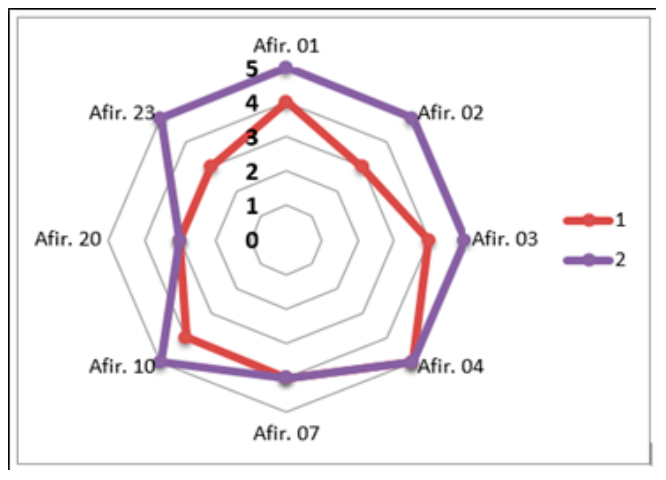

Figura 5. Muestra de las respuestas de los subgerentes de administración y finanzas (2) sobre las afirmaciones negativas

(8). Fuente: elaboración propia por parte de los autores.

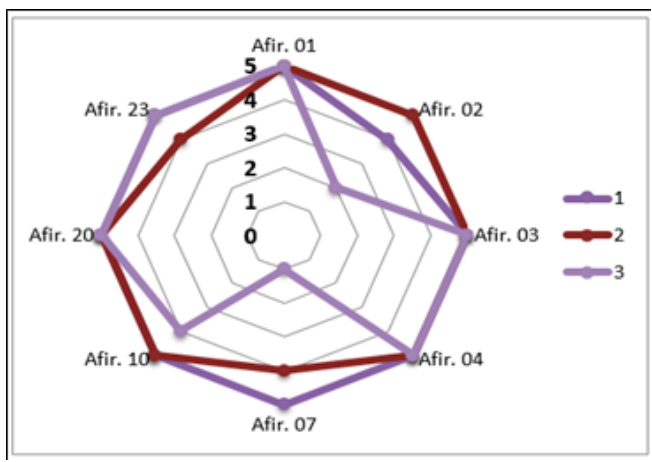

Figura 6. Muestra de las respuestas de los gerentes o subgerentes de contabilidad (3) sobre las afirmaciones negativas (8). Fuente: elaboración propia por parte de los autores.

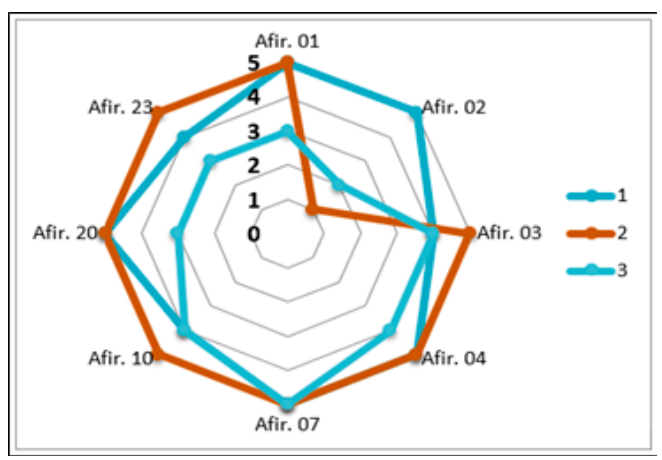

Figura 7. Muestra de las respuestas de los jefes de administración y finanzas (3) sobre las afirmaciones negativas

(8). Fuente: elaboración propia por parte de los autores.
Los resultados de las respuestas al instrumento aplicado fueron resumidos a nivel de coincidencias en las percepciones negativas de las distintas categorías de cargos que respondieron de las 21 empresas. Se encontraron respuestas a nivel de afirmaciones negativas por parte de los ejecutivos que se repiten y/o coinciden. Ver coincidencias con mayor frecuencia en las tablas 2 y 3 .

Tabla 2. Coincidencia de afirmaciones negativas

\begin{tabular}{cccccc}
\hline $\begin{array}{l}\text { No. } \\
\text { Afir. }\end{array}$ & G.A.F. & G.F.C. & $\begin{array}{l}\text { Sub } \\
\text { G.A.F. }\end{array}$ & $\begin{array}{l}\text { Sub } \\
\text { G.C. }\end{array}$ & J.A.F. \\
\hline 1 & $\mathrm{X}$ & & & $\mathrm{X}$ & $\mathrm{X}$ \\
\hline 2 & $\mathrm{X}$ & & $\mathrm{X}$ & $\mathrm{X}$ & $\mathrm{X}$ \\
\hline 7 & $\mathrm{X}$ & & & $\mathrm{X}$ & \\
20 & $\mathrm{X}$ & $\mathrm{X}$ & $\mathrm{X}$ & & $\mathrm{X}$ \\
23 & $\mathrm{X}$ & & $\mathrm{X}$ & & $\mathrm{X}$ \\
\hline
\end{tabular}

Fuente: elaboración propia por parte de los autores.

Tabla 3. Afirmaciones negativas del instrumento de mayor coincidencia

\begin{tabular}{|c|c|}
\hline No. & Afirmación del instrumento \\
\hline 1 & $\begin{array}{l}\text { Las funciones del Área/Dpto. de contabilidad no } \\
\text { contribuyen a las decisiones de la gerencia debido } \\
\text { a que la información que genera esta área es } \\
\text { insuficiente. }\end{array}$ \\
\hline 2 & $\begin{array}{l}\text { Las funciones del Área/Dpto. de contabilidad se } \\
\text { limitan solo a la contabilidad de la organización. }\end{array}$ \\
\hline 7 & $\begin{array}{l}\text { Las funciones del Área/Dpto. de contabilidad } \\
\text { ponen 'obstáculo' a la gerencia en momentos en } \\
\text { que se necesitan tomar decisiones rápidas, ya que } \\
\text { en el desempeño de la función de la contabilidad } \\
\text { deben hacerse gestiones específicas en los } \\
\text { sistemas contables en los tiempos legales o en } \\
\text { fechas de cierre límite. }\end{array}$ \\
\hline 20 & $\begin{array}{l}\text { La gerencia observa que las funciones del Área/ } \\
\text { Dpto. de contabilidad solo se limitan a cumplir con } \\
\text { normativas contables específicas y no es capaz de } \\
\text { agregar otras actividades a esta función. }\end{array}$ \\
\hline 23 & $\begin{array}{l}\text { Las funciones del Área/Dpto. de contabilidad } \\
\text { son consideradas como un "mal necesario" para } \\
\text { la gerencia debido a que, si bien es cierto que } \\
\text { esta área se ocupa del control de la información } \\
\text { de la organización, sus funciones hacen 'lento' el } \\
\text { proceso de toma de decisiones para la gerencia. }\end{array}$ \\
\hline
\end{tabular}

Fuente: elaboración propia por parte de los autores. 


\section{Discusión}

La percepción negativa de la administración puede originarse en una falta de conocimiento y/o experiencia en el uso, relevancia y aplicabilidad que se puede obtener a partir de la información que se origina desde los sistemas contables. Asimismo, puede que la administración no detecte las oportunidades del uso de información a partir de la contabilidad para construir entre otros: informes de gestión contable, financiera, tributaria, de operaciones u otro tipo de reportes requeridos.

En este sentido la percepción por parte de la administración se limita a depender de la información que proporcionan los estados financieros. Falta una visión más amplia que incorpore a la contabilidad como un medio de hacer gestión dentro de la organización, que sea capaz de elaborar información adicional ya sea financiera o no financiera y que agregue valor a la toma de decisiones de la administración. Los autores de esta investigación coinciden con lo expuesto por Buelvas y Mejía (2014) a la hora de tomar decisiones, pues no solo consideran a la contabilidad como la única fuente de información financiero-contable sino como información complementaria que proviene de otros sistemas de información de la organización.

Finalmente el análisis descriptivo de este trabajo de investigación indica una tendencia hacia la especialización en el ámbito de la gestión contable (no solo administrativa y financiera). La administración de la organización requiere que sus profesionales del área/departamento de contabilidad vayan más allá de las cifras de los estados financieros, por lo tanto es un desafío hacer que las cifras de la contabilidad se transformen en herramientas que generen información útil para tomar decisiones en distintos ámbitos de la organización.

\section{Conclusión}

Los resultados de este trabajo indican, en general, una percepción negativa. Los ejecutivos responden que la función de la contabilidad en las decisiones de la organización no contribuye a las decisiones de la gerencia y/o solo se limita a la contabilidad de las mismas o a cumplir solo normativas contables específicas y no agrega otras actividades a esta función dentro de la organización.

La percepción de estos ejecutivos coincide en que esta función de la contabilidad pone obstáculo a la gerencia en momentos que se necesita tomar decisiones rápidas, además es considerada como un mal necesario para la gerencia. Sin embargo la administración reconoce que las funciones que se llevan a cabo en esta área pueden controlar y administrar información de la organización, pero luego agregan que estas funciones de la contabilidad hacen lento el proceso de toma de decisiones para la gerencia.

\section{Referencias}

Amstrong, D. (1966). La percepción y el mundo físico. Madrid, España: Tecnos.

Arias Castilla, C. (2006). Enfoques teóricos sobre la percepción que tienen las personas. Horizontes Pedagógicos, 8(1), 9-22.

Arnheim, R. (1971). El pensamiento visual. Buenos Aires, Argentina: Universitaria de Buenos Aires.

Balmes, J. (1848). Curso de filosofía elemental. París, Francia: Garnier Hermanos.

Buelvas, C. y Mejía, G. (2014). El papel de la contabilidad de gestión en el sistema de información contable y su incidencia en la rentabilidad de las empresas. Panorama Económico, 22, 91-108.

Cañibano, L. (2004). Información financiera y gobierno de la empresa. Revista Internacional Legis de Contabilidad y Auditoría, 19, 157-236.

Carrizo, W. y León, S. (2007). ¿Qué realidad representa la contabilidad? Pecvnia, 5, 17-27. 
Dueñas, N. (2007). La teoría subjetiva del valor en contabilidad: comentarios sobre la valoración con base en las emociones. Innovar, 17(30), 145-152.

Freeman, R. (1983). Stockholders and Stakeholders: A New Perspective on Corporate Governace. California Management Review, 15(3), 88-106.

García, M. (2004). Fundamentos y normativas de la contabilidad. Valparaíso, Chile: LexisNexis.

Hernández, R., Fernández, C. y Baptista, M. (2010). Metodología de la investigación. Ciudad de México, México: McGraw-Hill.

Monterrey, J. (1998). Un recorrido por la contabilidad positiva. Revista Española de Financiación y Contabilidad, XXVII(95), 427-467.

Pérez, M. (2017). La aplicación de los gráficos radiales en el análisis contable del sector pesquero en Cataluña: gráfico radial y análisis contable. Revista Internacional de Economía y Gestión de las Organizaciones, 1(2), 98-128.

Zeff, S. (1985). Evolución de la teoría contable. La investigación empírica. Contaduría, 6, 25-53. 